\title{
REFLEXIVITY OF TENSOR PRODUCTS OF LINEAR TRANSFORMATIONS
}

\author{
WING SUET LI AND ELIZABETH STROUSE
}

(Communicated by Palle E. T. Jorgensen)

\begin{abstract}
Let $A$ and $B$ be linear transformations on finite-dimensional Hilbert space. We characterize the reflexivity of $A \otimes B$ in terms of certain characteristics of $A$ and $B$.
\end{abstract}

\section{INTRODUCTION}

Let $\mathscr{H}_{1}$ and $\mathscr{H}_{2}$ be separable, complex, Hilbert spaces, and suppose that $T_{1}$ and $T_{2}$ are bounded linear operators acting on $\mathscr{H}_{1}$ and $\mathscr{H}_{2}$, respectively. Then the tensor product $T_{1} \otimes T_{2}$ is a bounded linear operator acting on $\mathscr{H}_{1} \otimes \mathscr{H}_{2}$, and one may ask to what degree the structure of $T_{1} \otimes T_{2}$ is determined by that of $T_{1}$ and $T_{2}$. For instance, it was shown in [2] that the spectrum $\sigma\left(T_{1} \otimes T_{2}\right)$ is determined by the equation

$$
\sigma\left(T_{1} \otimes T_{2}\right)=\sigma\left(T_{1}\right) \sigma\left(T_{2}\right)=\left\{\lambda \mu: \lambda \in \sigma\left(T_{1}\right), \mu \in \sigma\left(T_{2}\right)\right\} .
$$

Also, if $T_{i}$ is similar to $U_{i}$ via the invertible operator $X_{i}, i=1,2$, then $T_{1} \otimes T_{2}$ is similar to $U_{1} \otimes U_{2}$ by virtue of the equation

$$
\left(X_{1} \otimes X_{2}\right)\left(T_{1} \otimes T_{2}\right)\left(X_{1} \otimes X_{2}\right)^{-1}=U_{1} \otimes U_{2},
$$

and so the similarity invariants of $T_{1} \otimes T_{2}$ are completely determined by those of $T_{1}$ and $T_{2}$. However, this information alone does not enable us to answer all questions concerning the structure of $T_{1} \otimes T_{2}$ in terms of the structure of $T_{1}$ and $T_{2}$. For example, one may ask whether the reflexivity of $T_{1} \otimes T_{2}$ follows from that of $T_{1}$ and (or) that of $T_{2}$. Very recently it was shown that in the infinite-dimensional case (see [5]) there exists $T_{1}$ and $T_{2}$ reflexive such that $T_{1} \otimes T_{2}$ is not reflexive. One is led to ask whether this type of "pathology" is possible in the finite-dimensional case. We show here that it is not. We present in addition (Theorem 3.1) necessary and sufficient conditions for the reflexivity of the tensor product of two operators. This characterization of the tensor product is an easy consequence of the Deddens-Fillmore theorem [4] and a result from linear algebra concerning Jordan forms of tensor products.

Received by the editors January 19, 1993 and, in revised form, June 1, 1993.

1991 Mathematics Subject Classification. Primary 47A80.

Key words and phrases. Tensor product, reflexivity.

The first author was partially supported by the National Science Foundation. The results of this paper were obtained while the first author was a visitor at the University of Bordeaux. 
Results concerning the Jordan form of tensor products were first obtained in the 1930s by Aitkin, Roth, and Littlewood [1, 8, 6]. Some gaps in the original proofs were filled in more recently by Marcus and Robinson [7] and by Brualdi [3]. These results were rediscovered independently by the authors. We present an elementary proof in $\S 4$.

\section{NOTATION AND PRELIMINARIES}

Let $\mathscr{B}(\mathscr{H})$ be the algebra of all bounded linear operators on a complex Hilbert space $\mathscr{H}$, and let $\mathscr{A}$ be a subalgebra of $\mathscr{B}(\mathscr{H})$. We define $\operatorname{Lat}(\mathscr{A})$ to be the lattice of invariant subspaces of the family $\mathscr{A}$ and $\operatorname{Alg}(\operatorname{Lat}(\mathscr{A}))$ to be the subalgebra of $\mathscr{B}(\mathscr{H})$ of operators which leave invariant all elements of $\operatorname{Lat}(\mathscr{A})$. We say that $\mathscr{A}$ is a reflexive algebra if $\mathscr{A}=\operatorname{Alg}(\operatorname{Lat}(\mathscr{A}))$. We say that an operator $T \in \mathscr{B}(\mathscr{H})$ is reflexive if the weakly closed algebra generated by $T$ and the identity operator $I$ is reflexive.

Now, suppose that $\mathscr{H}$ is finite dimensional. For each $\lambda$ in $\mathscr{C}$ and $n$ in $\mathscr{N}$ (the set of positive integers) let $J(\lambda, n)$ be the $n \times n$ complex Jordan block matrix with eigenvalue $\lambda$ (with 1's above the main diagonal). We denote by $N_{n}$ the matrix $J(0, n)$, the nilpotent matrix with degree of nilpotence equal to $n$. Let $\theta$ be a fixed linear ordering of $\mathscr{C}$. An $m \times m$ complex matrix $A$ will be said to be in Jordan form provided $A$ is a direct sum of Jordan blocks, say $A=\bigoplus_{i=1}^{k} J\left(\lambda_{i}, n_{i}\right)$, in which the distinct terms of the sequence $\left\{\lambda_{1}, \ldots, \lambda_{k}\right\}$ appear as in the ordering $\theta$ and the Jordan blocks corresponding to the same eigenvalue appear with increasing size. In the following, we denote by $\mathscr{H}_{n}$ an $n$-dimensional complex Hilbert space equipped with a fixed (ordered) orthonormal basis $\varepsilon_{n}=\left\{e_{1}^{n}, \ldots, e_{n}^{n}\right\}$, and we say that an operator on $\mathscr{U}_{n}$ is in Jordan form when its matrix with respect to $\varepsilon_{n}$ is in Jordan form. Clearly each operator $T$ acting on $\mathscr{H}_{n}$ is similar to exactly one operator in Jordan form on $\mathscr{H}_{n}$ which we denote by $J(T)$.

Definition 2.1. Let $A \in \mathscr{B}(\mathscr{H})$, and let $\lambda$ be an eigenvalue of $A$. We define:

- $n_{\lambda}^{A}$ to be the size of the largest Jordan block belonging to $\lambda$,

- $m_{\lambda}^{A}$ to be the size of the second largest Jordan block belonging to $\lambda$ ( $m_{\lambda}^{A}=0$ if $A$ has only one Jordan block belonging to $\lambda$ ),

- $S_{A}$ to be the set of all nonzero eigenvalues of $A$ associated with a one-dimensional eigenspace, and,

- $T_{A}$ to be the set of all nonzero eigenvalues $\lambda$ of $A$ such that $n_{\lambda}^{A}-m_{\lambda}^{A}>$ 1 .

We say that $\lambda$ has the Deddens-Fillmore property for $A$ if $n_{\lambda}^{A}-m_{\lambda}^{A} \leq 1$.

We note that the Deddens-Fillmore theorem characterizes reflexive linear operators on finite-dimensional Hilbert space as exactly those operators $A$ such that for each eigenvalue $\lambda$ of $A, n_{\lambda}^{A}-m_{\lambda}^{A} \leq 1$. Thus, if $A$ is reflexive, $T_{A}=\varnothing$.

Now, suppose that $A$ and $B$ are linear operators acting on the Hilbert spaces $\mathscr{H}_{m}$ and $\mathscr{H}_{n}$, respectively. In order to determine the reflexivity of $A \otimes B$, we need to know the relationship between the Jordan form of $A \otimes B$ and that of $A$ and of $B$. Since $A \otimes B$ is similar to the matrix $J(A) \otimes J(B)$, we may assume that $A$ and $B$ are in Jordan form. Since, by definition, the matrices $J(A)$ and $J(B)$ relative to the orthonormal bases $\left\{e_{k}^{m}\right\}_{k=1}^{m}$ and $\left\{e_{k}^{n}\right\}_{k=1}^{n}$ for $\mathscr{H}_{m}$ and $\mathscr{H}_{n}$ 
have the form

$$
J\left(\lambda_{1}, i_{1}\right) \oplus J\left(\lambda_{2}, i_{2}\right) \oplus \cdots \oplus J\left(\lambda_{s}, i_{s}\right)
$$

and

$$
J\left(\mu_{1}, j_{1}\right) \oplus J\left(\mu_{2}, j_{2}\right) \oplus \cdots \oplus J\left(\mu_{t}, j_{t}\right),
$$

respectively, it is clear that the matrix of $J(A) \otimes J(B)$ with respect to the orthonormal basis $\left\{e_{1}^{m} \otimes e_{1}^{n}, e_{1}^{m} \otimes e_{2}^{n}, \ldots, e_{1}^{m} \otimes e_{n}^{n}, \ldots, e_{m}^{m} \otimes e_{n}^{n}\right\}$ will have the form

$$
\bigoplus_{1 \leq k \leq s, 1 \leq l \leq t}\left(J\left(\lambda_{k}, i_{k}\right) \otimes J\left(\mu_{l}, j_{l}\right)\right)
$$

Thus, the following theorem contains the necessary information.

Theorem 2.2 ([1, 3, 6-8]). Let $n$ and $m$ be positive integers.

(i) Let $m \leq n$. The matrix $J(0, m) \otimes J(0, n)=N_{m} \otimes N_{n}$ has Jordan form

$$
\bigoplus_{i=1}^{m-1}(J(0, i) \oplus J(0, i)) \oplus\left(J(0, m) \otimes I_{n-m+1}\right) .
$$

(ii) For $\mu \neq 0, J(0, m) \otimes J(\mu, n)$ has Jordan form $\bigoplus_{i=1}^{n} J(0, m)$. Similarly, for $\lambda \neq 0, J(\lambda, m) \otimes J(0, n)$ has Jordan form $\bigoplus_{i=1}^{m} J(0, n)$.

(iii) Let $m \leq n$. For $\lambda, \mu \neq 0, J(\lambda, m) \otimes J(\mu, n)$ has Jordan form

$$
\bigoplus_{j=0}^{j=m-1}(J(\lambda \mu, n-m+1+2 j))
$$

Proofs of Theorem 2.2 have appeared in [1, 3, 6-8]. The authors rediscovered this theorem recently and present a new proof of it in $\S 4$.

\section{THE MAIN THEOREM}

Let $n_{\lambda}^{A}, m_{\lambda}^{A}, S_{A}$, and $T_{A}$ be defined as in Definition 2.1. We present necessary and sufficient conditions for the reflexivity of $A \otimes B$.

Definition 3.1. Let $T$ be a linear operator on a finite-dimensional Hilbert space $H$. We say that $A$ is of type $p$ if $A$ is similar to a matrix of the form $J(\lambda, 1) \oplus A_{0}$ where $\lambda \neq 0$ and $\sigma\left(A_{0}\right)=\{0\}$.

Theorem 3.2. Let $A$ and $B$ be linear operators on finite-dimensional complex Hilbert spaces $\mathscr{H}_{1}$ and $\mathscr{H}_{2}$, respectively, where $\operatorname{dim} \mathscr{H}_{1}$ and $\operatorname{dim} \mathscr{H}_{2}$ are both greater than 1. Suppose that neither $A$ and $B$ is of 'type $p$ '. Then $A \otimes B$ is not reflexive if and only if

$$
\exists_{\lambda_{0} \in S_{A} \cup T_{A}, \mu_{0} \in S_{B} \cup T_{B}} \text { such that } n_{\lambda_{0}}^{A}+n_{\mu_{0}}^{B}-1>\max \left\{M_{\lambda_{0}, \mu_{0}}^{A, B}, 1\right\}
$$

where $M_{\lambda_{0}, \mu_{0}}^{A, B}=\left\{n_{\lambda}^{A}+n_{\mu}^{B}: \lambda \in \sigma(A), \quad \mu \in \sigma(B), \lambda \mu=\lambda_{0} \mu_{0}, \quad(\lambda, \mu) \neq\right.$ $\left.\left(\lambda_{0}, \mu_{0}\right)\right\}$.

Proof. First, suppose that (1) holds. Let $\lambda_{0}$ and $\mu_{0}$ be such that $n_{\lambda_{0}}^{A}+n_{\mu_{0}}^{B}-1>$ $\max \left\{M_{\lambda_{0}, \mu_{0}}^{A, B}, 1\right\}$, and set $\gamma=\lambda_{0} \mu_{0}$. We note that $\gamma \neq 0$. Since (1) holds, Theorem 2.1(iii) implies that

$$
n_{\gamma}^{A \otimes B}=n_{\lambda_{0}}^{A}+n_{\mu_{0}}^{B}-1
$$


and that

$$
m_{\gamma}^{A \otimes B}=n_{\lambda_{0}}^{A}+n_{\mu_{0}}^{B}-3 .
$$

Thus $\gamma$ does not satisfy the Deddens-Fillmore condition for $A \otimes B$, and $A \otimes B$ is not reflexive.

Next, suppose that (1) does not hold and that $\gamma$ does not satisfy the DeddensFillmore condition for $A \otimes B$. If $\gamma \neq 0$, then $\gamma=\lambda \mu$ where

$$
n_{\gamma}^{A \otimes B}=n_{\lambda}^{A}+n_{\mu}^{B}-1 \text {. }
$$

Since (1) does not hold, we may assume without loss of generality that $\lambda \in$ $\sigma(A) \backslash\left(S_{A} \cup T_{A}\right)$. Then $\lambda$ satisfies the Deddens-Fillmore condition for $A$, and $m_{\lambda}^{A}>0$. But, in this case

$$
m_{\gamma}^{A \otimes B} \geq m_{\lambda}^{A}+n_{\mu}^{B}-1
$$

and so $n_{\lambda}^{A}-m_{\lambda}^{A} \geq n_{\gamma}^{A \otimes B}-m_{\gamma}^{A \otimes B}>1$, contradicting the fact that $\lambda$ satisfies the Deddens-Fillmore condition. Thus every nonzero eigenvalue of $A \otimes B$ satisfies the Deddens-Fillmore condition.

So, suppose that $0 \in \sigma(A \otimes B)$. There are three possible cases:

(i) $0 \in \sigma(A), 0 \notin \sigma(B)$;

(ii) $0 \in \sigma(B), 0 \notin \sigma(A)$;

(iii) $0 \in \sigma(A) \cap \sigma(B)$.

The first two cases are clearly equivalent.

In case (i) we have

$$
n_{0}^{A \otimes B}=m_{0}^{A \otimes B}=n_{0}^{A} .
$$

To see this, let $M=\max _{\mu \in \sigma(B)} n_{\mu}^{B}$. If $M>1$, (2) follows directly from Theorem 2.2(ii). If $M=1$, then, since $\operatorname{dim} \mathscr{H}_{2}>1, B$ has at least two eigenvalues, and so (2) once again follows from Theorem 2.2(ii). Thus, in cases (i) and (ii), 0 satisfies the Deddens-Fillmore condition.

So, suppose we are in case (iii) and that $\sigma(A)=\sigma(B)=\{0\}$. Then, by Theorem 2.2(i), $n_{0}^{A \otimes B}=N=\min \left\{n_{0}^{A}, n_{0}^{B}\right\}$ and, as above, we may assume $N>1$. In this case, if $n_{0}^{A} \neq n_{0}^{B}, m_{0}^{A \otimes B}=N$, and if $n_{0}^{A}=n_{0}^{B}, m_{0}^{A \otimes B}=N-1$. Now, suppose $\sigma(A) \neq\{0\}$. Then, if $\sigma(B)=\{0\}$, then $n_{0}^{A \otimes B}=m_{0}^{A \otimes B}=n_{0}^{B}$, using the fact that $A$ is not of type $p$. If $\sigma(B) \neq\{0\}$, then, using the fact that neither $A$ nor $B$ is of type $p$, with Theorem 2.2(i), (ii), we see that $n_{0}^{A \otimes B}=m_{0}^{A \otimes B}=\max \left\{n_{0}^{A}, n_{0}^{B}\right\}$. In each case, 0 satisfies the Deddens-Fillmore condition, and so $A \otimes B$ is reflexive.

The condition for matrices of type $p$ to be reflexive are slightly different:

(I) If $A$ and $B$ are both of type $p$, then $A \otimes B$ is reflexive if and only if

$$
n_{0}^{B} \leq \max \left\{m_{0}^{B}, n_{0}^{A}\right\}+1 \quad \text { and } n_{0}^{A} \leq \max \left\{m_{0}^{A}, n_{0}^{B}\right\}+1
$$

(II) If one of the two matrices, say $A$, is of type $p$ and $B$ is not, then $A \otimes B$ is reflexive if and only if all nonzero $\lambda$ in the spectrum of $B$ satisfy the Deddens-Fillmore condition and $n_{0}^{B} \leq \max \left\{m_{0}^{B}, n_{0}^{A}\right\}+1$.

We present some corollaries detailing the more interesting consequences. 
Corollary 3.3. Let $A$ and $B$ be linear operators on finite-dimensional complex Hilbert spaces $\mathscr{H}_{1}$ and $\mathscr{H}_{2}$, respectively, where $\operatorname{dim} \mathscr{H}_{1}$ and $\operatorname{dim} \mathscr{H}_{2}$ are both greater than 1 .

(i) If $S_{A} \cup T_{A}=\varnothing \Rightarrow A \otimes B$ is reflexive for all $B$ not of 'type $p$ '.

(ii) If $A$ and $B$ are both reflexive, then $A \otimes B$ is reflexive.

(iii) If $A$ is reflexive and $S_{A}=\varnothing$, then $A \otimes B$ is reflexive for all $B$.

(iv) If $\sigma(A)=\{0\}$, then $A \otimes B$ is reflexive for all $B$ not of 'type $p$ '.

Corollary 3.4. Let $T$ be a finite rank operator on a Banach space $E$. Let $E_{1} \oplus E_{2}$ be a direct sum decomposition of $E$ such that $E_{1}$ is finite dimensional, $E_{1} \in$ Lat $T$, and $E_{2} \subseteq \operatorname{ker} T$. Then $T$ is a reflexive operator if and only if $T_{\mid E_{1}}$ is a reflexive operator. Thus (i)-(iv) of Corollary 3.3 hold for finite rank operators $A$ and $B$.

Proof. Suppose that $T_{\mid E_{1}}$ is a reflexive operator. Let $S \in \operatorname{Alg}(\operatorname{Lat}(T))$. Then $S_{\mid E_{2}} \in \operatorname{Alg}\left(\operatorname{Lat}\left(0_{E_{2}}\right)\right)$, so $S_{\mid E_{2}}=\lambda I_{E_{2}}$. Let $v \in E_{2}$, and set $E_{1}^{\prime}=\operatorname{span}\left(E_{1},\{v\}\right)$ and $E_{2}^{\prime}=$ the complement in $E$ of $E_{1}^{\prime}$. Since $T_{\mid E_{1}}$ is reflexive, the DeddensFillmore theorem implies that $T_{\mid E_{1}^{\prime}}$ is reflexive. Thus $S_{\mid E_{1}^{\prime}}=q(T)$, and $S_{\mid E_{2}^{\prime}}=$ $\lambda_{E_{2}^{\prime}}$ with $q(0)=\lambda$. So $S=q(T)$, $\operatorname{Alg}(\operatorname{Lat}(T))$ is composed entirely of polynomials in $T$, and $T$ is reflexive. On the other hand, if $T$ is reflexive, the fact that Lat $T=$ Lat $T_{\mid E_{1}} \oplus$ Lat $0_{E_{2}}$ implies immediately that $T_{\mid E_{1}}$ is reflexive.

\section{Proof of Theorem 2.2}

It is well known and easy to derive that, if $A$ is any linear operator on finite-dimensional Hilbert space, then

$$
\begin{aligned}
& \operatorname{dim}\left(\operatorname{ker}(A-\lambda)^{k}\right)-\operatorname{dim}\left(\operatorname{ker}(A-\lambda)^{k-1}\right) \\
& =\text { number of Jordan blocks belonging to } \lambda \text { of size } \geq k \\
& \quad \text { in the Jordan decomposition of } A .
\end{aligned}
$$

So, let $T_{m, n}=J(\lambda, m) \otimes J(\mu, n)-\lambda \mu I_{m n}$. For $\lambda=\mu=0, \operatorname{rank}\left(T_{m, n}^{k}\right)=$ $(m-k)(n-k)$; for $\lambda=0$ and $\mu \neq 0, \operatorname{rank}\left(T_{m, n}^{k}\right)=(m-k) n$; while for $\lambda \neq 0$ and $\mu=0, \operatorname{rank}\left(T_{m, n}^{k}\right)=(n-k) m$ (easily calculated by multiplying the block matrices and noticing that the rank of the resulting matrix is exactly equal to the number of nonzero rows). (i) and (ii) follow directly.

The proof of (iii) is more complicated. First, note that the matrix of $T_{m, n}$ with respect to an appropriate basis is of the form

$$
T_{m, n}=\left(\begin{array}{ccccc}
\lambda N_{n} & J(\mu, n) & 0 & \ldots & 0 \\
0 & \lambda N_{n} & J(\mu, n) & \ddots & \vdots \\
\vdots & \ddots & \ddots & \ddots & 0 \\
\vdots & \ddots & \ddots & \lambda N_{n} & J(\mu, n) \\
0 & \ldots & \ldots & 0 & \lambda N_{n}
\end{array}\right)
$$


Let

$$
X=\left(\begin{array}{ccccc}
(J(\mu, n))^{m-1} & 0 & \cdots & \cdots & 0 \\
0 & (J(\mu, n))^{m-2} & \ddots & & \vdots \\
\vdots & \ddots & \ddots & \ddots & \vdots \\
\vdots & & \ddots & (J(\mu, n)) & 0 \\
0 & \ldots & \ldots & 0 & I_{n}
\end{array}\right)
$$

It is easy to see that $X$ is invertible and that $C_{m, n}=X^{-1}\left(T_{m, n}\right) X$ is of the form

$$
C_{m, n}=\left(\begin{array}{ccccc}
\lambda N_{n} & I_{n} & 0 & \ldots & 0 \\
0 & \lambda N_{n} & I_{n} & \ddots & \vdots \\
\vdots & \ddots & \ddots & \ddots & 0 \\
\vdots & \ddots & \ddots & \lambda N_{n} & I_{n} \\
0 & \ldots & \ldots & 0 & \lambda N_{n}
\end{array}\right)
$$

Notice that $\operatorname{rank}\left(T_{m, n}\right)^{k}=\operatorname{rank}\left(C_{m, n}\right)^{k}$ and that

$$
\left(C_{m, n}\right)^{k}=\left(\begin{array}{cccc}
a_{0} N_{n}^{k} & a_{1} N_{n}^{k-1} & \ldots & a_{m-1} N_{n}^{k-m+1} \\
0 & \ddots & \ddots & \vdots \\
\vdots & \ddots & a_{0} N_{n}^{k} & a_{1} N_{n}^{k-1} \\
0 & \ldots & 0 & a_{0} N_{n}^{k}
\end{array}\right)
$$

where $a_{i}=\lambda^{k-i}\left(\begin{array}{c}k \\ i\end{array}\right)$ and we define $N_{n}^{-j}=0$ for $j>0$ and $\left(\begin{array}{c}k \\ i\end{array}\right)=0$ if $i>k$.

We use two rather technical lemmas to determine $\operatorname{rank}\left(C_{n, m}\right)^{k}$.

Lemma 4.1. Let $p, k, r \in \mathscr{Z}, k \geq p \geq 0, p+r \geq 0$. Let $A$ be the matrix with coordinates

$$
a_{i j}=\left(\begin{array}{c}
k \\
p-i+j
\end{array}\right) \quad(i, j=0,1, \ldots, p+r)
$$

where $\left(\begin{array}{l}k \\ l\end{array}\right)$ is defined to be zero for $l>k$ or $l<0$. Then

$$
\operatorname{det} A=\frac{\left(\begin{array}{l}
k \\
p
\end{array}\right)\left(\begin{array}{l}
k+1 \\
p+1
\end{array}\right) \cdots\left(\begin{array}{c}
k+p+r \\
2 p+r
\end{array}\right)}{\left(\begin{array}{c}
k+r \\
p+r
\end{array}\right)\left(\begin{array}{c}
k+r-1 \\
p+r-1
\end{array}\right) \cdots\left(\begin{array}{c}
k-p \\
0
\end{array}\right)}
$$

Proof. Notice that, for notational convenience, our matrix has a '0th' row and a '0th' column.

Let $A_{k, p, r}$ be the matrix defined in (4). We claim that

$$
\operatorname{det}\left(A_{k, p, r}\right)=\frac{\left(\begin{array}{l}
k \\
p
\end{array}\right)}{\left(\begin{array}{l}
k+r \\
p+r
\end{array}\right)} \operatorname{det} A_{k+1, p+1, r-2}
$$

which proves the lemma (by induction on $p+r$ ).

Given a matrix $A_{k, p, r}$ to see that the claim is true, just subtract $\left(\frac{a_{i, 0}}{a_{i-1,0}}=\right.$ $\left.\frac{p-i+1}{k-p+i}\right)$ times the $(i-1)$ st row from the $i$ th row, and put the result into the $i$ th row. Since

$$
\left(\begin{array}{c}
k \\
p-i+j
\end{array}\right)-\frac{p-i+1}{k-p+i}\left(\begin{array}{c}
k \\
p-i+j+1
\end{array}\right)=\frac{j}{k-p+i}\left(\begin{array}{c}
k+1 \\
p-i+j+1
\end{array}\right),
$$


the new matrix has entries

$$
b_{i j}= \begin{cases}a_{i, j}, & i=0,0 \leq j \leq p+r \\
0, & 1 \leq i \leq p+r, j=0 \\
\frac{j}{k-p+i}\left(\begin{array}{c}
k+1 \\
p-i+j+1
\end{array}\right), & 1 \leq i, j \leq p+r\end{cases}
$$

so that

$$
\operatorname{det} A_{k, p, r}=\left(\begin{array}{c}
k \\
p
\end{array}\right) \operatorname{det}\left[b_{i j}\right]_{i, j=1}^{p+r}=\frac{\left(\begin{array}{l}
k \\
p
\end{array}\right)}{\left(\begin{array}{c}
k+r \\
p+r
\end{array}\right)} \operatorname{det} A_{k+1, p+1, r-2} .
$$

Lemma 4.2. Let $m, n, k \in \mathscr{N}, m \leq n$. Then

$$
\operatorname{Rank}\left(T_{m, n}\right)^{k}= \begin{cases}\frac{m(n-k)}{\frac{(m+n-k)^{2}}{4}} & \text { if } k \leq m-n, \\ & \text { if } n+m-1>k>n-m \\ \text { and } m+n-k \text { even }, & \\ & \text { if } n+m-1>k>n-m \\ & \text { and } m+n-k \text { odd }, \\ 0 & \text { if } k \geq m+n-1 .\end{cases}
$$

Proof. We divide the matrix $\left(C_{m, n}\right)^{k}$ into $m$ blocks of $n$ rows and denote by $\operatorname{ROW}(I, J)$ the $J$ th row of the $I$ th block. The rows can then be divided into $m+n-2$ equivalence classes $\left\{R_{p}\right\}_{p=2}^{m+n}$ where

$$
R_{p}=\{R(I, J): I+J=p\} .
$$

We note that $R_{p}$ has $\alpha_{p}$ elements where

$$
\alpha_{j}= \begin{cases}j-1, & j=2, \ldots, m, \\ m, & j=m+1, \ldots, n+1, \\ m+n+1-j, & j=n+1, \ldots, m+n,\end{cases}
$$

and that the nonzero rows of $\left(C_{m, n}\right)^{k}$ are precisely those rows in the equivalence classes $R_{2}, R_{3}, \ldots, R_{m+n-k}$.

Similarly, by dividing $\left(C_{m, n}\right)^{k}$ into $m$ blocks of $n$ columns we define $\operatorname{COL}(I, J)$ and the equivalence classes $C_{p}(p=2,3, \ldots, m+n)$. (As before $C_{p}$ has $\alpha_{p}$ elements.) It is easily seen that the nonzero columns of the elements of the class $R_{j}$ are precisely the elements of the class $C_{j+k}$. Thus $\left\{R_{p}\right\}_{p=2}^{m+n}$ is a pairwise orthogonal set and

$$
\operatorname{rank}\left(\left(C_{m, n}\right)^{k}\right)=\sum_{j=2}^{m+n-k} \operatorname{dim}\left(\operatorname{span}\left(R_{j}\right)\right) I
$$


Now, let

$$
\begin{aligned}
p_{j} & =\min \left(\alpha_{j}, \alpha_{j+k}\right) \\
& =\min \left(\text { number of elements of } R_{j}, \text { number of elements of } C_{j+k}\right) .
\end{aligned}
$$

Since the matrix with rows equal to the elements of $R_{j}$ contains a submatrix of form (4) with $p+r=p_{j}$, we get

$$
\operatorname{Rank}\left(\left(C_{m, n}\right)^{k}\right)=\sum_{j=2}^{m+n-k} p_{j}
$$

It is easily seen that

$$
p_{j}= \begin{cases}\alpha_{j}, & 2 \leq j \leq \gamma \\ \alpha_{j+k}, & \gamma<j \leq m+n-k\end{cases}
$$

where

$$
\gamma= \begin{cases}n+1-k & \text { for } k \leq n-m, \\ \frac{m+n-k}{2}+1 & \text { for } k>n-m \text { and } m+n-k \text { even } \\ \frac{m+n-k+1}{2} & \text { for } k>n-m \text { and } m+n-k \text { odd }\end{cases}
$$

and now a simple computation finishes the proof.

We will now prove Theorem 2.2(iii). Using Lemma 4.2 we see that, if we set $\kappa_{k}=\operatorname{dim}\left(\operatorname{ker}\left(T_{m, n}\right)^{k}\right)-\operatorname{dim}\left(\operatorname{ker}\left(T_{m, n}\right)^{k-1}\right)$, then

$$
\kappa_{k}= \begin{cases}m & \text { if } 1 \leq k \leq n-m+1, \\ m+\left[\frac{k-(n-m)}{2}\right] & \text { if } n-m+2 \leq k \leq m+n-1, \\ 0 & \text { if } k>n+m-1,\end{cases}
$$

where $[r]=$ the greatest integer less than $r$ for each real number $r$. Thus, using equation (3),

$$
\text { \# of blocks of size } k= \begin{cases}0 & \text { if } k<n-m+1, \\ 1 & \text { if } n-m+1 \leq k \leq n+m-1 \\ & \text { and } k-(n-m) \text { odd } \\ 0 & \text { if } n-m+1 \leq k \leq n+m-1 \\ & \text { and } k-(n-m) \text { even, } \\ 0 \quad \text { if } k>n+m-1,\end{cases}
$$

which finishes the proof.

\section{ACKNOWLEDGMENT}

The authors would like to thank the referee and I. Chalandar for pointing out an error in the original statement of Theorem 3.2. 


\section{BIBLIOGRAPHY}

1. A. C. Aitken, The normal form of compound and induced matrices, Proc. London Math. Soc. 38 (1934), 354-376.

2. A. Brown and C. Pearcy, Spectra of tensor products of operators, Proc. Amer. Math. Soc. 17 (1966), 162-166.

3. R. A. Brualdi, Combinatorial verification of the elementary divisors of tensor products, Linear Algebra Appl. 71 (1985), 31-47.

4. J. A. Deddens and P. A. Fillmore, Reflexive linear transformations, Linear Algebra Appl. 1 (1975), 89-93.

5. D. Larson and W. R. Wogen, Reflexivity properties of $T \oplus 0$, J. Funct. Anal. 92 (1990), 448-467.

6. D. E. Littlewood, On induced and compound matrices, Proc. London Math. Soc. 40 (1936), 370-380.

7. M. Marcus and H. Robinson, Elementary divisors of tensor products, Comm. ACM 18 (1975), 36-39.

8. W. E. Roth, On direct product matrices, Bull. Amer. Math. Soc. 40 (1934), 461-468.

Department of Mathematics, Indiana University, BLoomington, Indiana 47405

Current address: School of Mathematics, Georgia Institute of Technology, Atlanta, Georgia 30080

E-mail address: li@math.gatech.edu

U.F.R. De MathematiQues, Université de BordeauX I, 351 Cours de la Liberation, 33405 TALENCE, France

E-mail address: strouse@ceremab.u-bordeaux.fr 